\title{
Semi-Analytical Calculation of the Armature Reaction in Slotted Tubular Permanent Magnet Actuators
}

\author{
Bart L. J. Gysen, Koen J. Meessen, Johannes J. H. Paulides, and Elena A. Lomonova \\ Department of Electrical Engineering, Electromechanics, and Power Electronics, Eindhoven University of Technology \\ Eindhoven $5600 \mathrm{MB}$, The Netherlands
}

\begin{abstract}
This paper considers the semi-analytical field calculation of the armature reaction in brushless tubular permanent magnet actuators with rectangular slots. The tubular actuator is considered to have an infinite length and Fourier analysis is used to describe the current density distribution. The field solution is obtained by solving the Maxwell equations in the constant boundary value problem consisting of the airgap and the slot. The results are verified with finite element software, and analysis limitations and inaccuracies are addressed.
\end{abstract}

Index Terms-Analytical solution, permanent-magnet machines, stator slots.

\section{INTRODUCTION}

$\mathbf{L}$ INEAR and tubular actuators are ever more frequently used in the industry since they offer various advantages in comparison to the rotary to linear counterparts. Tubular actuators have excellent servo characteristics, virtually zero attraction force, and a higher force density than flat linear machines. In order to obtain an optimal design subject to various specifications, accurate modeling of the behavior of the actuator is inevitable. Various techniques exist for predicting the electromagnetic behavior of the brushless tubular actuator, including the magnetic equivalent circuit (MEC) networks [1], the Schwarz-Christoffel (SC) conformal mapping technique [2], the finite element method (FEM) [3], and the semi-analytical approach [4]. The latter one is the most preferred for optimization, due to the high accuracy and fast computational time. Although linear iron with infinite permeability is assumed in the semi-analytical framework, a space mapping optimization routine [1] could give a solution to include the nonlinear behavior. Various publications exist on the analytical solution of the magnetic field resulting from the permanent magnets [5]-[8], and force calculation is obtained by means of the Lorentz equation where the windings are modeled as current sheets. This approximation is valid for slotless machines; however, in the case of a slotted machine, this simplification leads to inaccuracies of the force profile and inductance calculation. Therefore, a semi-analytical approach for calculation of the magnetic field resulting from the stator windings including slotting effect is proposed in this paper. Rectangular slots and only a full pitch winding with one slot per pole per phase is considered; however, the same technique can be applied for other winding topologies. In Section II, the assumptions of the model are addressed, Section III describes the method and solution of the electromagnetic field in the airgap and the slot, and the results are verified with the FEM in Section IV. Furthermore, a brief discussion of the appearing inaccuracies due to the proposed method is given.

\section{AsSUMPTIONS}

To be able to apply the semi-analytical framework, several assumptions are made in order to describe the electromagnetic

Digital Object Identifier 10.1109/TMAG.2008.2001662

Color versions of one or more of the figures in this paper are available online at http://ieeexplore.org.

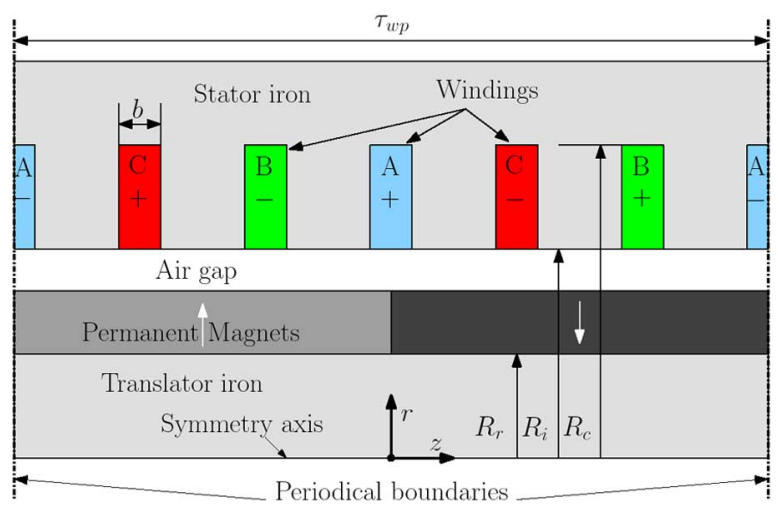

Fig. 1. Illustration of a single coil pitch of the cross section of a tubular permanent magnet actuator with full pitch windings and one slot per pole per phase.

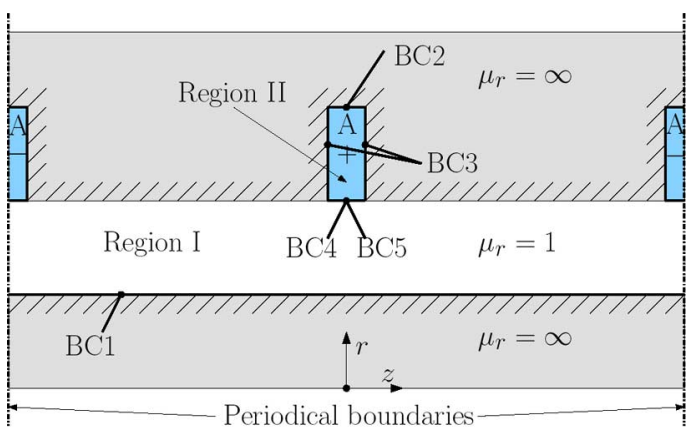

Fig. 2. Division in regions of a single segment with the various boundary conditions (BCs).

field solution. The axis symmetry of the tubular actuator allows the use of a 2-D cylindrical coordinate system. In the analysis, end effects are excluded and a single coil pitch with periodical boundary conditions is considered as shown in Fig. 1. Only the field solution due to the stator windings will be derived; hence, the permanent magnets are modeled as air since their permeability is close to unity. For multiphase systems, as, for example, the three-phase system of Fig. 1, superposition is applied to obtain the total field solution with the assumption that slotting effects of neighboring slots are neglected. This assumption means that only the slot due to the phase under consideration can be taken into account, as shown in Fig. 2. 


\section{Semi-AnaLytical Model}

The field equations are expressed in terms of the magnetic vector potential, $\mathbf{A}$, which is given by [9]

$$
\mathbf{B}=\nabla \times \mathbf{A}
$$

where the magnetic field, $\mathbf{B}$, due to the symmetrical nature, only has components in the $r$-and $z$-direction, $B_{r}$ and $B_{z}$, respectively, and the vector potential has only one component in the $\theta$-direction, $A_{\theta}$, resulting in

$$
\begin{aligned}
\mathbf{B} & =B_{r}(r, z) \mathbf{e}_{r}+B_{z}(r, z) \mathbf{e}_{z} \\
B_{r} & =-\frac{\partial A_{\theta}}{\partial z} \\
B_{z} & =\frac{\partial}{r \partial r}\left(r A_{\theta}\right)
\end{aligned}
$$

where $\mathbf{e}_{r}$ and $\mathbf{e}_{z}$ are unit vectors in the r- and z-direction, respectively. The current density of region II, J, can be written as a Fourier series, using the coordinate system in Fig. 2, given by

$$
\begin{aligned}
\mathbf{J} & =J_{\theta} \mathbf{e}_{\theta} \\
J_{\theta} & =\sum_{n=1}^{\infty} J_{n} \cos \left(m_{n} z\right) \\
J_{n} & =\frac{4 J_{A}}{(2 n-1) \pi} \sin \left(\frac{m_{n} b}{2}\right) \\
m_{n} & =\frac{2(2 n-1) \pi}{\tau_{w p}}
\end{aligned}
$$

since the current density flows in the $\theta$-direction with an amplitude $J_{A}$ and spatial frequencies $m_{n}$, where $n$ is the harmonic number. The field equations in terms of the magnetic vector potential are then given by the Laplace equation in region I and the Poisson equation in region II for the 2-D-coordinate system which represent Ampere's law for regions I and II

$$
\begin{aligned}
\frac{\partial}{\partial r}\left(\frac{1}{r} \frac{\partial r A_{\mathrm{I}_{\theta}}}{\partial r}\right)+\frac{\partial^{2} A_{\mathrm{I}_{\theta}}}{\partial z 2} & =0 \\
\frac{\partial}{\partial r}\left(\frac{1}{r} \frac{\partial r A_{\mathrm{II}_{\theta}}}{\partial r}\right)+\frac{\partial^{2} A_{\mathrm{II}_{\theta}}}{\partial z^{2}} & =-\mu_{0} J_{\theta}
\end{aligned}
$$

where the indices I and II correspond to regions I and II, respectively, and $\mu_{0}$ is the permeability of vacuum. Furthermore, since the relative permeability of copper is almost equal to unity, the constitutive relations are given by

$$
\begin{aligned}
\mathbf{B}_{\mathrm{I}} & =\mu_{0} \mathbf{H}_{\mathrm{I}} \\
\mathbf{B}_{\mathrm{II}} & =\mu_{0} \mathbf{H}_{\mathrm{II}}
\end{aligned}
$$

with $\mathbf{H}$ the magnetic field intensity. The appropriate solution of the Laplace and Poisson equations for this problem can be written as

$$
\begin{aligned}
B_{\mathrm{I}_{r}} & =\sum_{n=1}^{\infty}\left(a_{1 n} \mathcal{B}_{\mathcal{I}_{1}}\left(m_{n} r\right)+b_{1 n} \mathcal{B}_{\mathcal{K}_{1}}\left(m_{n} r\right)\right) \sin \left(m_{n} z\right) \\
B_{\mathrm{I}_{z}} & =\sum_{n=1}^{\infty}\left(a_{1 n} \mathcal{B}_{\mathcal{I}_{0}}\left(m_{n} r\right)-b_{1 n} \mathcal{B}_{\mathcal{K}_{0}}\left(m_{n} r\right)\right) \cos \left(m_{n} z\right) \\
B_{\mathrm{II}_{r}} & =\sum_{k=1}^{\infty}\left(a_{2 k} \mathcal{B}_{\mathcal{I} 1}\left(p_{k} r\right)+b_{2 k} \mathcal{B}_{\mathcal{K}_{1}}\left(p_{k} r\right)\right)
\end{aligned}
$$

TABLE I

PARAMETERS OF THE MODEL

\begin{tabular}{|l|l|l|}
\hline Parameter & Value & Description \\
\hline$R_{r}$ & $5 \mathrm{~mm}$ & Outer radius of the translator iron \\
$R_{i}$ & $11 \mathrm{~mm}$ & Inner radius of the stator \\
$R_{c}$ & $25 \mathrm{~mm}$ & Outer radius of the coils \\
$b$ & $5 \mathrm{~mm}$ & Width of the slots \\
$\tau_{w p}$ & $80 \mathrm{~mm}$ & Coil pitch \\
$J_{A}$ & $5 \mathrm{~A} / \mathrm{mm}^{2}$ & Current density of phase A \\
$N$ & 70 & Maximum number of harmonics in region I \\
$K$ & 15 & Maximum number of harmonics in region II \\
\hline
\end{tabular}

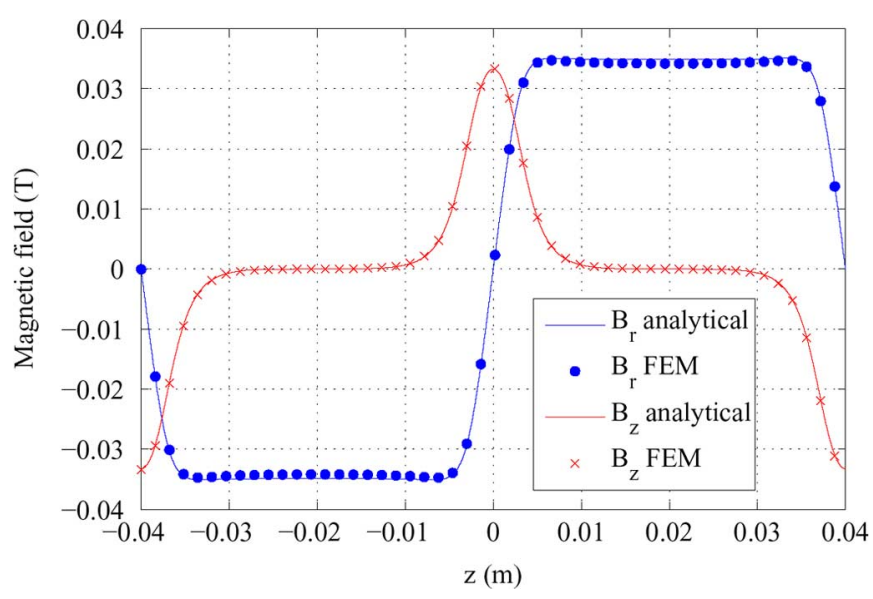

Fig. 3. Magnetic field distribution in the center of the airgap, $r=10.5 \mathrm{~mm}$.

$$
\begin{aligned}
& \times \sin \left(p_{k}\left(z+\frac{b}{2}\right)\right) \\
B_{\mathrm{II}_{z}}= & \sum_{k=1}^{\infty}\left(a_{2 k} \mathcal{B}_{\mathcal{I}_{0}}\left(p_{k} r\right)-b_{2 k} \mathcal{B}_{\mathcal{K}_{0}}\left(p_{k} r\right)\right) \\
& \times \cos \left(p_{k}\left(z+\frac{b}{2}\right)\right)-\mu_{0} J_{\theta} r+B_{0}
\end{aligned}
$$

where $\mathcal{B}_{\mathcal{I}_{i}}(x)$ and $\mathcal{B}_{\mathcal{K}_{i}}(x)$ are modified Bessel functions of the first and second kind, respectively, with order $i$, and $p_{k}=(k 2 \pi / b)$ are the spatial frequencies of the slot with harmonic number $k$. The coefficients $a_{1 n}, b_{1 n}, a_{2 k}, b_{2 k}$, and $B_{0}$ are determined by solving the set of boundary conditions (BCs), as indicated in Fig. 2:

- $\mathrm{BC} 1:\left.B_{\mathrm{I}_{z}}\right|_{r=R_{r}}=0$

- $\mathrm{BC} 2:\left.B_{\mathrm{II} z}\right|_{r=R_{c}}=0$

- $\mathrm{BC} 3:\left.B_{\mathrm{II}_{r}}\right|_{z= \pm(b / 2)}=0$

- BC4: $\left.B_{\mathrm{I}_{r}}\right|_{r=R_{i},|z|<(b / 2)}=\left.B_{\mathrm{II}_{r}}\right|_{r=R_{i},|z|<(b / 2)}$

- BC5: $\left.H_{\mathrm{I}_{z}}\right|_{r=R_{i},|z|<(b / 2)}=\left.H_{\mathrm{II}_{z}}\right|_{r=R_{i},|z|<(b / 2)}$

where the solution is given in the Appendix.

\section{VALIDATION OF Model ACCURACY BY FINITE ELEMENT ANALYSIS}

The semi-analytical solution is obtained in the airgap (region I) and slot (region II) for the parameters given in Table I.

First, the semi-analytical solution is compared with the solution of a FEM model created in Flux 2-D [10], which has the same assumptions as the semi-analytical model, described in Section II and Fig. 2. Only a finite number of harmonics, $N$ and $K$ as given in Table I, can be calculated due to numerical limitations. Figs. 3 and 4 show clearly good agreement between the FEM and semi-analytical models. However, care 


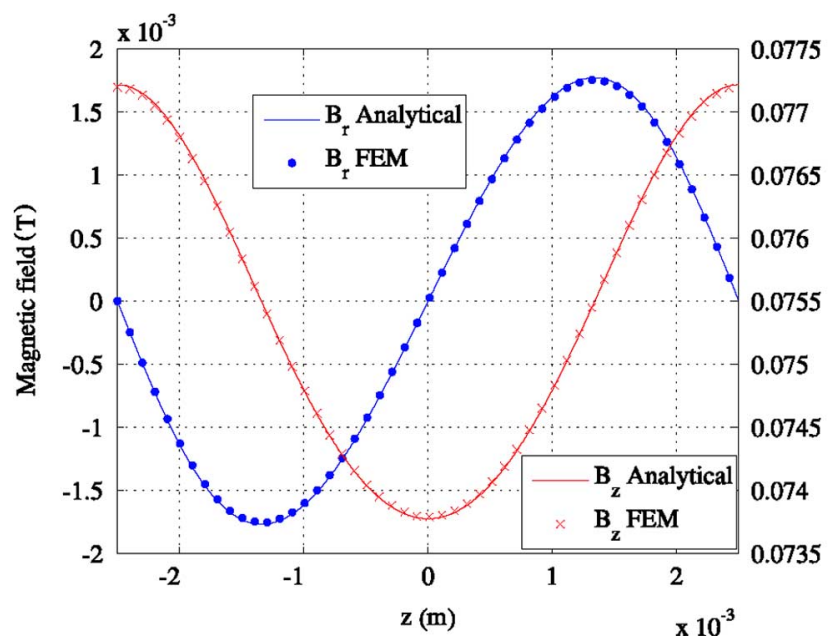

Fig. 4. Magnetic field distribution in the slot, $r=13 \mathrm{~mm}$.

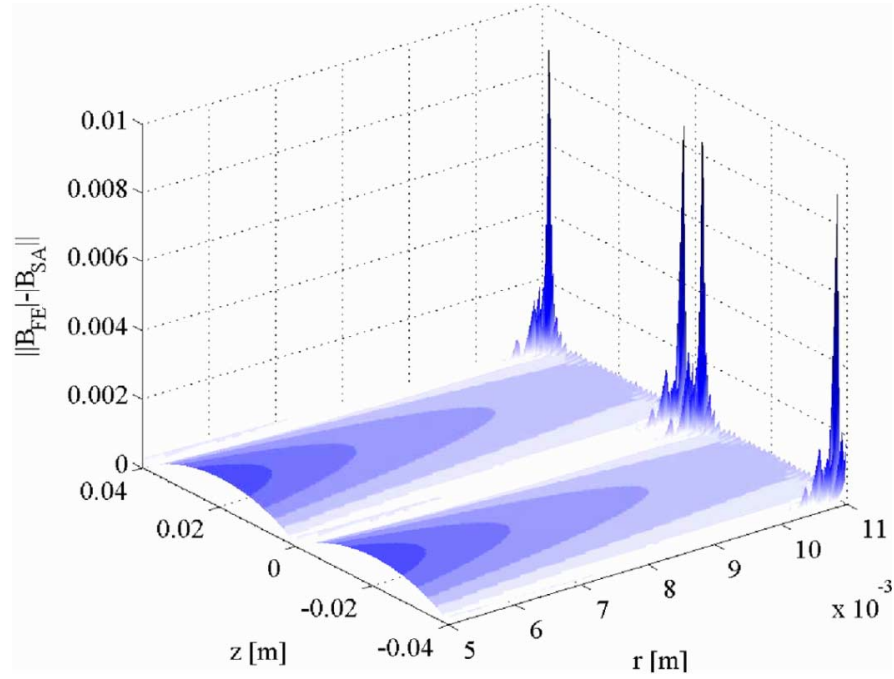

Fig. 5. Difference between the FEM and semi-analytical model for region I, $5<r<11 \mathrm{~mm}$.

should be taken when using the semi-analytical solution. Therefore, consider the difference between the magnitude of the magnetic field of the FEM model, $\left|B_{\mathrm{FE}}\right|$, and the semi-analytical model, $\left|B_{\mathrm{SA}}\right|$. This difference is plotted for regions I and II in Figs. 5 and 6 , respectively, where the error of region II is only plotted for $r<12 \mathrm{~mm}$ since for higher radii the error is less than $1 \%$ of the magnitude. In both Figs. 5 and 6 , at the boundary of region I and II, relatively high errors (around 10\%) occur. This error is predominantly resulting from the "mode matching" problem, as further discussed in [11]. Because, at that boundary, two field solutions of different spatial frequencies should be equal, given by the evaluated boundary equations $\mathrm{BC} 4$ and $\mathrm{BC} 5$

$$
\begin{aligned}
\sum_{n=1}^{\infty} B_{\mathrm{I}_{r_{n}}} \sin \left(m_{n} z\right)= & \sum_{k=1}^{\infty} B_{\mathrm{II}_{r_{k}}} \sin \left(p_{k}\left(z+\frac{b}{2}\right)\right) \\
\sum_{n=1}^{\infty} B_{\mathrm{I}_{z_{n}}} \cos \left(m_{n} z\right)= & \sum_{k=1}^{\infty} B_{\mathrm{II}_{z_{k}}} \cos \left(p_{k}\left(z+\frac{b}{2}\right)\right) \\
& -\mu_{0} J_{\theta} R_{i}+B_{0} .
\end{aligned}
$$

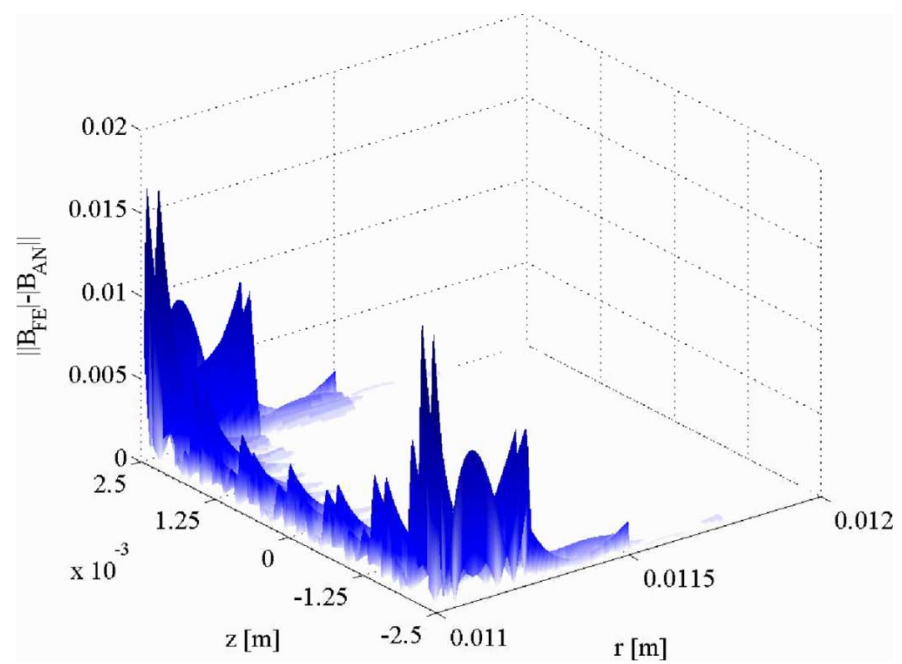

Fig. 6. Difference between the FEM and semi-analytical model for region II where $r<12 \mathrm{~mm}$.

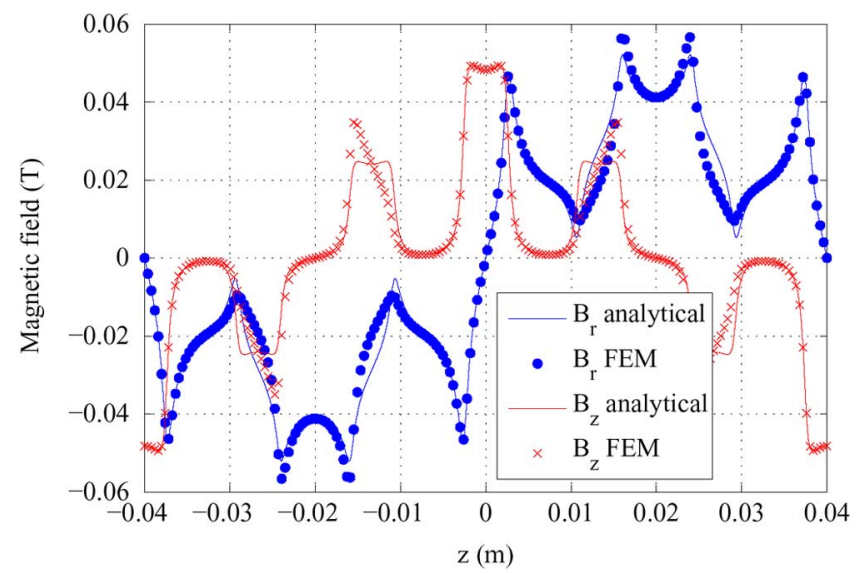

Fig. 7. Magnetic field distribution in region I for the three-phase system, $r=$ $10.5 \mathrm{~mm}$.

The Bessel functions increase exponentially with the harmonic number and only a finite number of harmonics, $N$ and $K$ for region I and II, respectively, can be calculated due to numerical limitations, which leads to the inaccuracy indicated in Figs. 5 and 6. However, it can be observed that this error decays very fast for a change in the radius and because for force calculation only the magnetic field solution in the center of the airgap is necessary, an accurate force prediction is still obtained.

Second, the total field distribution for the three-phase winding topology is calculated and compared with a FEM model, as indicated in Fig. 1, but without the permanent magnets. From the solution shown in Fig. 7, it can be observed that higher errors appear since the total field solution is obtained by superposition of the individual field solutions of each phase where slotting effect of the other phases is excluded (Section II). However, still a more precise solution is obtained with respect to the current sheet model for a slotted stator. As this error does not predominantly appear in the slot region, the inductance calculation will still be accurate.

The computation time of the analytical method is around $0.05 \mathrm{~s}$, whereas the linear 2-D finite element calculation takes about $1 \mathrm{~s}$ for a mesh of 13000 elements. Regarding optimization algorithms, the effort is even larger since the analytical method 
can easily be integrated in the software used for optimization, e.g., Matlab.

\section{CONCLUSION}

A semi-analytical solution of the electromagnetic field distribution of the armature reaction is given for a brushless tubular permanent magnet actuator with a full pitch winding topology with one slot per pole per phase for rectangular slots. The solution is obtained by solving the Maxwell equations for the constant boundary value problem consisting of an airgap and slot region. If a multiphase system is considered, the total field distribution is approximated by superposition of the field distribution of the individual phases with the assumption that the slotting effect of the other phases is neglected. The solution has good agreement with the FEM; however, inaccuracies occur at the boundary of the airgap and slot region, and care should be taken when evaluating the magnetic field at that point. This field solution can be used to predict the inductance or the force profile in combination with the semi-analytical field solution resulting from the magnets.

\section{APPENDIX}

First define the following diagonal matrices:

$$
\begin{aligned}
\mathbf{C}_{1 N} & =\operatorname{diag}\left(\mathcal{B}_{\mathcal{I} 0}\left(m_{n} R_{r}\right)\right) \\
\mathbf{C}_{2 N} & =\operatorname{diag}\left(\mathcal{B}_{\mathcal{K} 0}\left(m_{n} R_{r}\right)\right) \\
\mathbf{C}_{3 N} & =\operatorname{diag}\left(\mathcal{B}_{\mathcal{I} 0}\left(m_{n} R_{i}\right)\right) \\
\mathbf{C}_{4 N} & =\operatorname{diag}\left(\mathcal{B}_{\mathcal{K} 0}\left(m_{n} R_{i}\right)\right) \\
\mathbf{C}_{5 N} & =\operatorname{diag}\left(\mathcal{B}_{\mathcal{I} 1}\left(m_{n} R_{i}\right)\right) \\
\mathbf{C}_{6 N} & =\operatorname{diag}\left(\mathcal{B}_{\mathcal{K} 1}\left(m_{n} R_{i}\right)\right) \\
\mathbf{C}_{7 K} & =\operatorname{diag}\left(\mathcal{B}_{\mathcal{I} 0}\left(p_{k} R_{i}\right)\right) \\
\mathbf{C}_{8 K} & =\operatorname{diag}\left(\mathcal{B}_{\mathcal{K} 0}\left(p_{k} R_{i}\right)\right) \\
\mathbf{C}_{9 K} & =\operatorname{diag}\left(\mathcal{B}_{\mathcal{I} 1}\left(p_{k} R_{i}\right)\right) \\
\mathbf{C}_{10 K} & =\operatorname{diag}\left(\mathcal{B}_{\mathcal{K} 1}\left(p_{k} R_{i}\right)\right) \\
\mathbf{C}_{11 K} & =\operatorname{diag}\left(\mathcal{B}_{\mathcal{I} 1}\left(p_{k} R_{c}\right)\right) \\
\mathbf{C}_{12 K} & =\operatorname{diag}\left(\mathcal{B}_{\mathcal{K} 1}\left(p_{k} R_{c}\right)\right)
\end{aligned}
$$

Further, define the following functions:

$$
\begin{gathered}
\varepsilon(k, n)= \begin{cases}\frac{2 k \tau_{w p}^{2} \sin \left(\frac{b(2 n-1) \pi}{\tau_{w p}}\right)}{\pi\left((2 n-1)^{2} b^{2}-k^{2} \tau_{w p}^{2}\right)}, & \text { for } m_{n} \neq p_{k} \\
\cos (k \pi), & \text { for } m_{n}=p_{k}\end{cases} \\
\eta(k, n)= \begin{cases}\frac{4(2 n-1) b^{2} \sin \left(\frac{b(2 n-1) \pi}{\tau_{w p}}\right)}{\pi\left((2 n-1)^{2} b^{2}-k^{2} \tau_{w p}^{2}\right)}, & \text { for } m_{n} \neq p_{k} \\
\frac{b}{\tau_{w p}} \cos (k \pi), & \text { for } m_{n}=p_{k}\end{cases} \\
\sigma(n)=\frac{8}{m_{n} \tau_{w p} \sin \left(\frac{b(2 n-1) \pi}{\tau_{w p}}\right) .}
\end{gathered}
$$

The boundary conditions result in a set of governing equations which can be written as

$$
\left[\begin{array}{l}
\mathbf{a}_{1 N} \\
\mathbf{b}_{1 N} \\
\mathbf{a}_{2 K} \\
\mathbf{b}_{2 K}
\end{array}\right]=\mathbf{E}^{-1} \mathbf{Y}
$$

where $\mathbf{a}_{1 N}, \mathbf{b}_{1 N}, \mathbf{a}_{2 K}$, and $\mathbf{b}_{2 K}$ are column vectors with elements $a_{1 n}, b_{1 n}, a_{2 k}$, and $b_{2 k}$, respectively. $\mathbf{E}$ and $\mathbf{Y}$ are given by

$$
\begin{aligned}
\mathbf{E} & =\left[\begin{array}{cccc}
\mathbf{C}_{1 N} & -\mathbf{C}_{2 N} & \mathbf{0} & \mathbf{0} \\
\mathbf{C}_{3 N} & -\mathbf{C}_{4 N} & -\boldsymbol{\eta}_{N K} \mathbf{C}_{7 K} & \boldsymbol{\eta}_{N K} \mathbf{C}_{8 K} \\
\mathbf{0} & \mathbf{0} & \mathbf{C}_{11 K} & \mathbf{C}_{12 K} \\
-\varepsilon_{K N} \mathbf{C}_{5 N} & -\varepsilon_{K N} \mathbf{C}_{6 N} & \mathbf{C}_{9 K} & \mathbf{C}_{10 K}
\end{array}\right] \\
\mathbf{0} & \\
\mathbf{Y} & =\left[\begin{array}{c}
-\mu_{0} R_{i} \mathbf{J}_{N}+\boldsymbol{\sigma}_{N} B_{0} \\
\mathbf{0} \\
\mathbf{0}
\end{array}\right]
\end{aligned}
$$

with $B_{0}=\mu_{0} J_{A} R_{c}$ and where $\varepsilon_{K N}$ is a $\mathrm{K} \times \mathrm{N}$ matrix consisting of elements $\varepsilon(n, k), \boldsymbol{\eta}_{N K}$ is a $\mathrm{N} \times \mathrm{K}$ matrix consisting of elements $\eta(k, n), \boldsymbol{\sigma}_{N}$ is a column vector consisting of elements $\sigma(n)$ and $\mathbf{J}_{N}$ is a column vector consisting of elements $J_{n}$.

\section{REFERENCES}

[1] L. Encica, J. J. H. Paulides, E. A. Lomonova, and A. J. A. Vandenput, "Electromagnetic and thermal design of a linear actuator using output polynomial mapping," in Proc. IEEE Ind. Appl. Soc. 41th Annu. Meeting, Oct. 2006, vol. 4, pp. 1919-1926.

[2] D. C. J. Krop, E. A. Lomonova, and A. J. A. Vandenput, "Application of Schwarz-Christoffel mapping to permanent-magnet linear motor analysis," IEEE Trans. Magn., vol. 44, no. 3, pp. 352-359, Mar. 2008.

[3] J. L. G. Janssen, J. J. H. Paulides, E. A. Lomonova, and A. J. A. Vandenput, "Cogging force reduction in tubular permanent magnet actuators," in Proc. IEEE Int. Electric Machines and Drives Conf. (IEMDC'07), Antalia, Turkey, May 2007, vol. 1, pp. 266-271.

[4] J. Wang, G. W. Jewell, and D. Howe, "A general framework for the analysis and design of tubular linear permanent magnet machines," IEEE Trans. Magn., vol. 35, no. 3, pp. 1986-2000, May 1999.

[5] J. Wang and D. Howe, "Tubular modular permanent-magnet machines equipped with quasi-halbach magnetized magnets-Part I: Magnetic field distribution, emf, and thrust force," IEEE Trans. Magn., vol. 41, no. 9, pp. 2470-2478, Sep. 2005.

[6] J. Wang, D. Howe, and G. W. Jewell, "Analysis and design optimization of an improved axially magnetized tubular permanent-magnet machine," IEEE Trans. Energy Convers., vol. 19, no. 2, pp. 289-295, Jun. 2004.

[7] J. Wang, D. Howe, and Y. Amara, "Armature reaction field and inductances of tubular modular permanent magnet machines," J. Appl. Phys., vol. 97, no. 10Q504, pp. 1-3, 2005.

[8] Z. J. Liu and J. T. Li, "Analytical solution of air-gap field in permanentmagnet motors taking into account the effect of pole transition over slots," IEEE Trans. Magn., vol. 43, no. 10, pp. 3872-3883, Oct. 2007.

[9] E. P. Furlani, Permanent Magnet and Electromechanical Devices. New York: Academic, 2001.

[10] FLUX2D 10.1 User's Guide. Cedrat, Meylan, France, 2007.

[11] W. S. Lee, W. R. Jones, and J. J. Campbell, "Convergence of numerical solutions of iris-type discontinuity problems," IEEE Trans. Microw. Theory Tech., vol. 19, no. 6, pp. 528-536, Jun. 1971.

Manuscript received March 03, 2008. Current version published December 17, 2008. Corresponding author: B. L. Gysen (e-mail: b.1.j.gysen@tue.nl). 